\title{
Asplenium patagonicum (Aspleniaceae, Pteridophyta) nueva especie para Chile
}

\section{Asplenium patagonicum (Aspleniaceae, Pteridophyta) new species from Chile}

\author{
Roberto Rodríguez Ríos* \& Rosario Guzmán-Marín
}

Departamento de Botánica, Facultad de Ciencias Naturales y Oceanográficas, Universidad de Concepción. Casilla 160-C, Concepción, Chile.

*rrodrigu@udec.cl

\begin{abstract}
A new Chilean species, Asplenium patagonicum (Aspleniaceae, Pteridophyta), on the basis of the collections of vascular plants of South Chile is described. This Chilean fern has been reclassified from Asplenium monanthes L., given their differences in geographic distribution and morphological features. While A. patagonicum occurs in South Chile, A. monanthes presents a tropical distribution. The latter also is clearly distinguished from A. patagonium given its smaller size and different shape in their pinna; small, non-linear, and deltoid rhizome scales, and shorter general size.
\end{abstract}

En el marco del proyecto Expedición Última Patagonia 2014 realizada por la Asociación franco-chileno de Centre Terre a la Isla Diego de Almagro de la Reserva Nacional Alacalufes, se hizo un estudio de la vegetación y recolección de plantas vasculares presentes en el lugar. La Isla Diego de Almagro ha sido poco intervenida bajo el punto de vista humano, formó parte de la antigua ruta de la población Kawésqar dedicada a la recolección de recursos marinos para la alimentación; entre los años 1914 y 1928 fue explorada para estudiar el potencial de explotación de caliza, sin embargo, la empresa minera no prosperó dejando en abandono las faenas y la infraestructura.

La zona norte de la isla se caracteriza por sus afloramientos rocosos principalmente de caliza y mármol, sobre los que crece un bosque dominado por Nothofagus betuloides (Mirb.) Oerst. y Nothofagus antarctica (G. Forst.) Oerst., en otros sectores se encuentran suelos de areniscas-lutitas, dejando depresiones donde se deposita agua, permitiendo el desarrollo de turberas de Astelia pumila (G. Forst.) Gaudich., matorrales bajos con pequeños bosques de Pilgerodendron uviferum (D. Don) Florin, con musgos y plantas vasculares en forma de cojín. En general, el ambiente de la isla presenta fuertes limitantes para el desarrollo de la vegetación, en especial las altas precipitaciones, las bajas temperaturas y los fuertes vientos fríos de occidente (Gajardo 1994).

Las características climáticas y geográficas han hecho de la isla una zona muy poco explorada, por consiguiente, la vegetación es poco conocida. Es de gran interés la flora de Pteridófitas, entre las que se han encontrado, por ejemplo, Asplenium dareoides Desv., Blechnum magellanicum
(Desv.) Mett., Hymenophyllum ferrugineum Colla, $H$. magellanicum (Desv.) Willd. ex Kunze, H. pectinatum Cav., H. peltatum (Poir.) Desv., H. secundum Hook. \& Grev., H. tortuosum Hook. \& Grev. Entre los helechos se registró a Asplenium monanthes L., una especie que tiene una amplia distribución en el mundo, la que comprende: África y Madagascar, varias islas del Atlántico (Azores, Madeira, Canarias, Tristan d'Acunha), Hawaii, América del Norte (USA, México), América Central (Guatemala, El Salvador, Honduras, Jamaica, Nicaragua, Costa Rica) y América del Sur (Venezuela, Colombia, Ecuador, Brasil, Perú, Bolivia, Uruguay, Argentina y Chile) (Tryon \& Stolze 1993, Rodríguez 1995).

Existen registros de la especie en la Argentina, donde Asplenium monanthes crece en las provincias del norte, pero no pasa al sur de la Provincia de Buenos Aires; desde allí, la distribución tiene un salto hacia el lado occidental de la Cordillera de los Andes pues aparece en el sur de Chile. Los materiales chilenos recolectados en los alrededores del Lago Ranco durante el siglo XIX han sido mencionados como Asplenium monanthemum (sinónimo de Asplenium monanthes L.) por Mettenius (1856) "55 b. var. Sori in utroque latere segmentorum. P1. chil. 769. Lagun. d. Ranco". J.W. Sturm (1858) se refiere a la misma planta anterior y dice: "Chile: in truncis arborum ad lacum Laguna de Ranco (Lechler pl. chil. N. 769)". Hooker (1860) se refiere al mismo taxón pero de otro recolector "Chili, Bridges, n. 808, at the base and on the trunks of trees. Laguna de Ranco". Por último, de acuerdo con Looser (1938) el ejemplar recolectado por Otto Philippi en Futrono cerca del Lago 
Ranco fue determinado por R.A. Philippi, quien agrega en la etiqueta del ejemplar "Asplenium longifolium Ph." pensando establecer una especie nueva que nunca publicó, y que sólo aparece mencionada por Gotschlich (1913) en una lista, sin una descripción. Los materiales encontrados más al sur de la Región de Los Lagos mantienen las mismas características que los ya mencionados.

Mediante un análisis de la morfología de las pinnas, de forma y del tamaño de las escamas del rizoma, de la distribución geográfica y del hábitat de esos materiales, se ha llegado a la conclusión que se trata de un nuevo taxón para la flora chilena.

\section{Asplenium patagonicum R.A. Rodr. \& R. Guzmán}

Plants terrestrial, rizome scales deltoid to lanceolate, to $3 \mathrm{~mm}$ long; leaves pinnate of 10-25 cm long and 1-2 cm broad; rachis glabrous, with remains of the pinnae basis, canaliculate abaxialy; pinnae of $0.5-1 \mathrm{~cm}$ long and $1-2 \mathrm{~cm}$ broad, quadrangular to round, oblique at the basis, subentire to crenate-serrate in the margin; sori 1-4 per pinna.

Typus: Provincia Última Esperanza. Archipiélago Madre de Dios, Isla Tarlton. 25 m s.m. (50²1'S - 75'22'W). 26- II2013. E. Teneb 792 (CONC 177963).

Sinónimo: Asplenium longifolium Phil. ex Gotschl., Bol. Mus. Nac. Chile 6(1): 347. 1913, nom. nud.

Planta terrestre, rizoma corto, erecto, con escamas débilmente clatradas, deltoide-lanceoladas de hasta $3 \mathrm{~mm}$ de longitud, de color castaño claro. Hojas fasciculadas, pinnadas, de 1025 x 1-2 cm. Pecíolo de 3-9 cm de longitud, de color castaño oscuro, lustroso. Lámina delgada, herbácea, gradualmente estrechada hacia los extremos. Raquis glabro, con los restos de las bases de las pinnas, canaliculado abaxial. Pinnas de 0,5-1 cm de longitud, casi sésiles, cuadrangulares a redondeadas, oblicuas en la base, ápice romo, subenteras o crenado-serradas en el margen; soros 1-4, indusio lateral, de color verde pálido, persistente (Figs. 1 A-C).

Planta endémica de Chile, conocida de las provincias de Cautín, Ranco, Osorno y Última Esperanza, donde crece entre 10 a $320 \mathrm{~m}$ de altitud (Fig. 2). Habita en el piso del bosque, sobre troncos caídos, escaso en grietas de rocas calizas, o en lugares rocosos y soleados.

Las especies de Asplenium se ubican en cuatro zonas de Chile bien definidas:

a) Isla de Pascua (A. obtusatum G. Forst. var. obtusatum y $A$. polyodon G. Forst. var. squamulosum (C. Chr.) R.A. Rodr.;

b) Archipiélago de Juan Fernández (A. macrosorum Bertero ex Colla y A. stellatum Colla);

c) Zona Norte, entre Arica y Coquimbo (A. gilliesii Hook., A. peruvianum Desv. y A. triphyllum C. Presl);

d) Zona Centro y Sur, entre Coquimbo y Magallanes ( $A$. dareoides Desv., A. obtusatum G. Forster var. sphenoides (Kunze) C. Chr. ex Skottsb., A. patagonicum R.A. Rodr. \& R. Guzmán y A. trilobum Cav.). Estas últimas se pueden separar mediante la siguiente clave:

1. Lámina simple, entera o trilobulada

A. trilobum

1'. Lámina dividida

2. Lámina 2-3-tripinnada .

A. dareoides

2'. Lámina pinnada

3. Pinnas mayores de $2 \mathrm{~cm}$ de longitud

3'. Pinnas menores de $1,5 \mathrm{~cm}$ de longitud

A. obtusatum var. sphenoides A. patagonicum

Materiales estudiados: CHILE. Región de la Araucanía, Provincia de Cautín, Río Imperial. X-1889. A. Boisson (SGO). Región de Los Ríos, Provincia de Ranco, Futrono, cerca del Lago Ranco. Jan. 1887. O. Philippi (SGO). Región de Los Lagos, Provincia de Osorno, Lago Puyehue, área de la desembocadura del río Pescadero. 200 m s.m. 26 -XII-1937. C. Rudolph (CONC, G, SGO); Lago Puyehue. 3-XII-1943. M.R. Espinosa (SGO). Región de Magallanes, Provincia Última Esperanza: Archipiélago Madre de Dios,
Isla Tarlton. $25 \mathrm{~m}$ s.m. $\left(50^{\circ} 21^{\prime} \mathrm{S}-75^{\circ} 22^{\prime} \mathrm{W}\right) .26-$ II-2013. E. Teneb 792 (Typus, CONC); Isla Madre de Dios. 44 m s.m. $\left(50^{\circ} 05^{\prime} \mathrm{S}-75^{\circ} 07^{\prime} \mathrm{W}\right) .16-\mathrm{I}-2010$. CEQUA-PNBO 1080 (CONC); Isla Guarello. 10 m s.m. (50²1'S - 75²0'W). 13-XI-1976. I. Moyano (CONC); Isla Guarello. 25-XII1958. E.J. Godley 788 (SGO). Isla Diego de Almagro. 320 m s.m. (51 $\left.22^{\circ} \mathrm{S}-75^{\circ} 04^{\prime} \mathrm{W}\right)$. 08-II-2014. R. Guzmán 27 (CONC). 

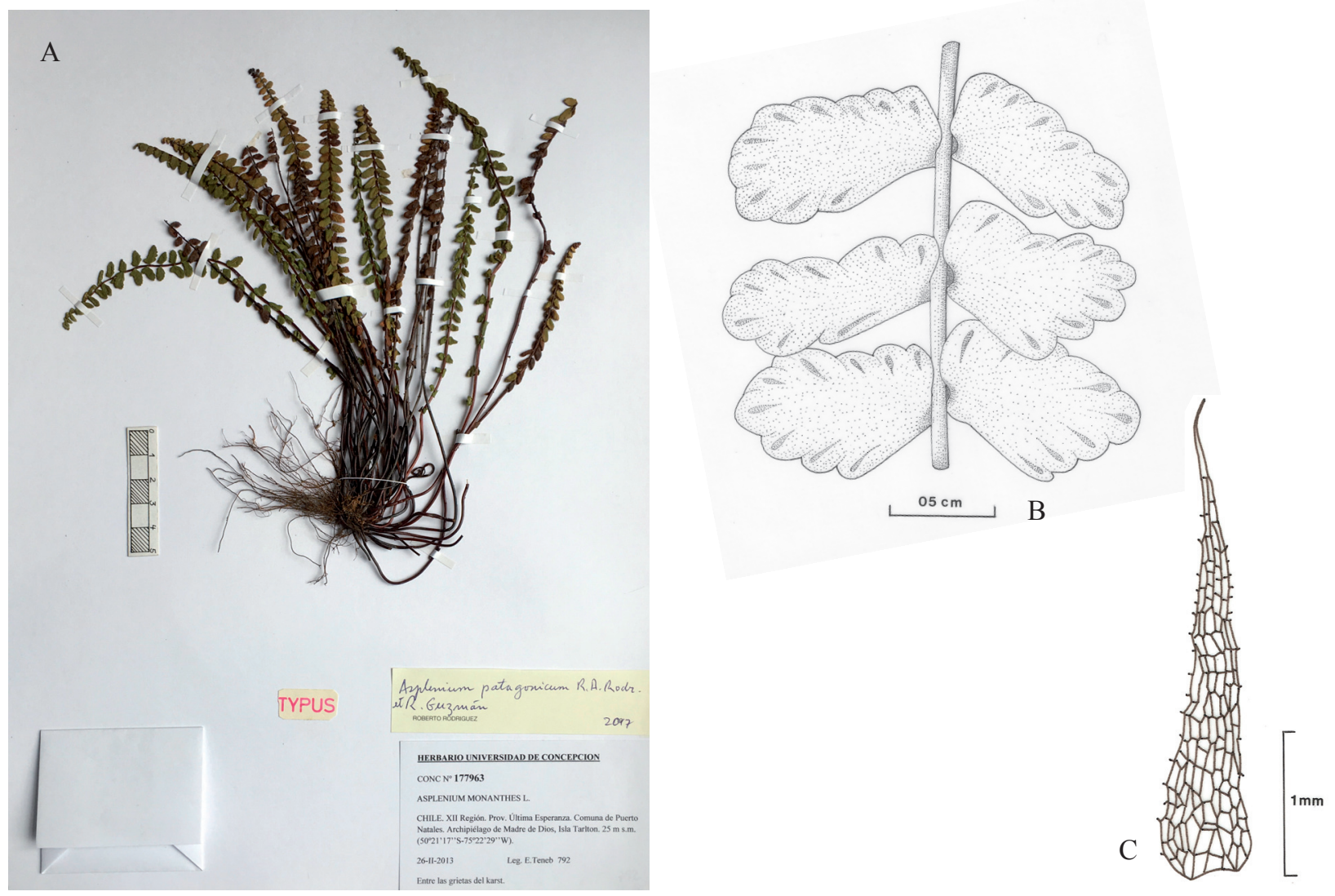

Figura 1. A. Typus de Asplenium patagonicum. B. Pinnas. C. Escama (CONC 177963). / A. Typus of Asplenium patagonicum. B. Pinnae. C. Scale (CONC 177963).

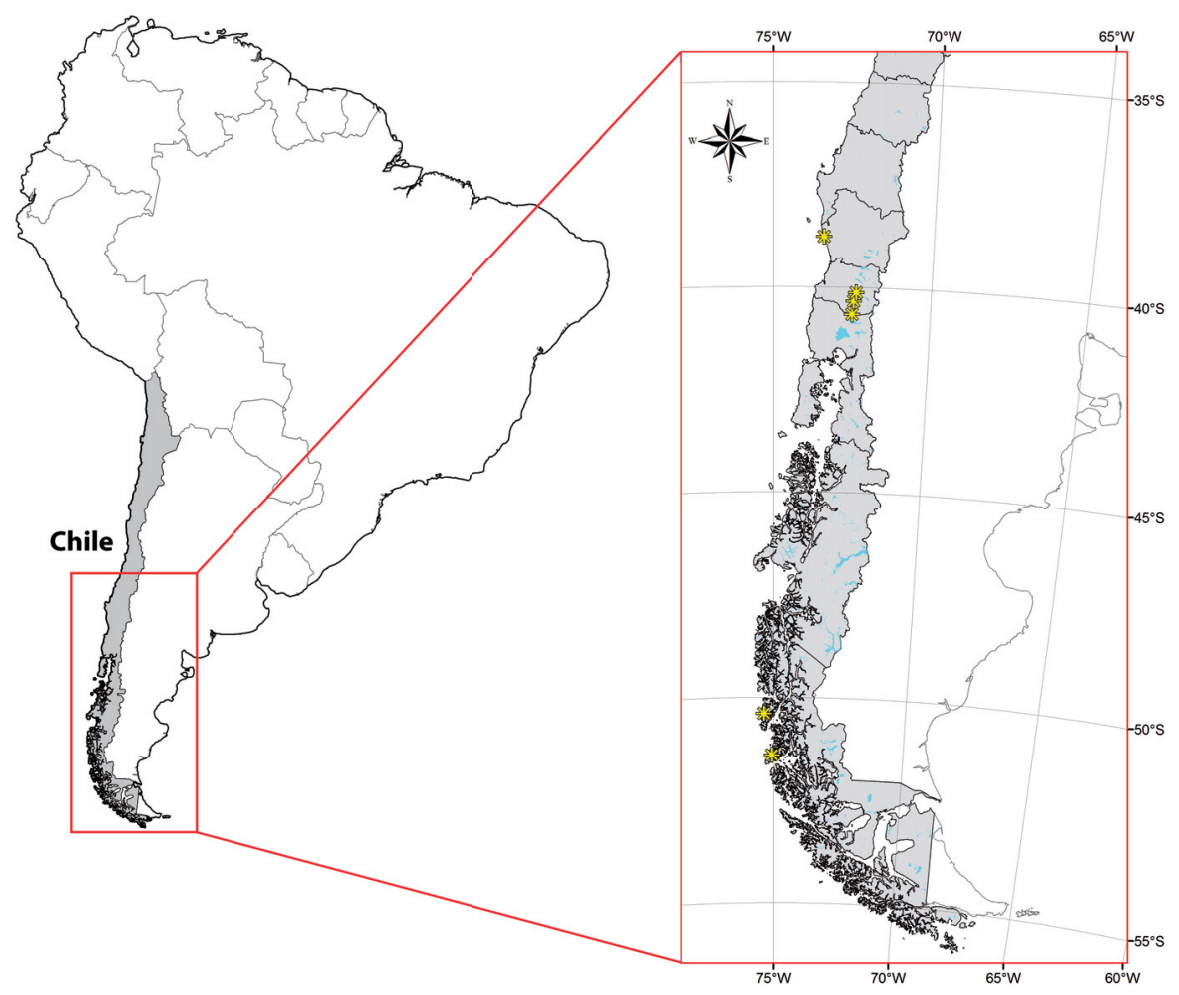

FIgURA 2. Mapa de distribución de Asplenium patagonicum. / Distribution map of Asplenium patagonicum. 


\section{AGRADECIMIENTOS}

Los autores agradecen al Proyecto Expedición Última Patagonia 2014 Centre Terre y a la Facultad de Ciencias Naturales y Oceanográficas de la Universidad de Concepción por el apoyo logístico en terreno en la Isla Diego de Almagro. Al proyecto del Fondo de Investigación del Bosque Nativo (CONAF) $\mathrm{N}^{\circ} 004 / 2016$. A Alicia Marticorena, curadora del Herbario CONC, por sus acertados consejos, a Gloria Rojas por poner a nuestra disposición los materiales del Herbario del Museo Nacional de Historia Natural (SGO). Agradecemos también a la Bióloga Diplomada Andrea Kiessling por toda la ayuda entregada en los distintos pasos para la preparación del manuscrito y a Pedro Guzmán quien preparó el mapa de distribución.

\section{REFERENCIAS}

Gajardo, R. 1994. La vegetación natural de Chile, clasificación y distribución geográfica. Editorial Universitaria, Santiago de Chile. 165 pp.

Gotschlich, B. 1913. Llanquihue i Valdivia. Boletín del Museo Nacional de Historia Natural 6(1): 7-626.

HoOKER, W.J. 1860. Species Filicum 3: 1-291.

Looser, G. 1938. El helecho Asplenium monanthes L. y su presencia en Chile. Revista Sudamericana de Botánica 5(3-4): 75-77.

Mettenius, G.H. 1856. Filices lechlerianae, chilenses ac peruanae. 1: 30 pp., 3 lám. Leipzig.

Rodríguez, R. 1995. Pteridophyta. En: C. Marticorena y R. Rodríguez (eds.). Flora de Chile. Vol. 1: 119-309. Ed. Universidad de Concepción, Concepción, Chile.

Sturm, J.W. 1858. Enumeratio plantarum vascularium cryptogamicarum chilensium. Abhandlungen der Naturhistorischen Gesellschaft zu Nürnberg. 1: 151-202.

Tryon, R.M., Stolze, R.G. 1993. Pterodophyta of Peru. Part. V. 18. Aspleniaceae -21 . Polypodiaceae. Fieldiana Botany, n.s. 32: 1-190.

Recibido: 06.10.2016

Aceptado: 29.03.2017 